\title{
Unterstützte Kommunikation für Menschen ohne Sprache Patienteninformation
}

\section{Für wen ist Unterstützte Kommu- nikation (UK) eine Hilfe? \\ $\nabla$}

Es gibt viele Gründe, warum eine Person nicht spricht, nur einen sehr kleinen Wortschatz hat oder nur schwer zu verstehen ist. Hierzu gehören angeborene und erworbene Beeinträchtigungen als auch

- eine geistige, motorische oder mehrfache Behinderung,

- eine Autismus-Spektrums-Störung,

- ein Schlaganfall, Hirntumor oder Schädel-Hirn-Trauma,

• eine degenerative Erkrankung.

\section{Was bewirkt UK?}

UK dient dem Anbahnen, Ergänzen oder Ersetzen von Lautsprache und ist für alle Altersgruppen relevant. Bei Kindern, die aufgrund einer Entwicklungsverzögerung nicht altersgemäß beginnen zu sprechen, kann der Einsatz von Gebärden oder Bildsymbolen parallel zur klassischen Sprachtherapie helfen, dass das Kind anfängt zu sprechen. Es erlebt z.B. über das Zeigen auf Bildkarten, dass es etwas durch diese Mitteilung bewirken kann, und erweitert seinen Wortschatz.

Manche Beeinträchtigungen führen auch dazu, dass der Wortschatz deutlich eingeschränkt ist oder die Person nur schwer zu verstehen ist. Nahe stehenden Personen mag es gelingen, das meiste zu verstehen, doch selbst dann kommt es zu frustrierenden Situationen, in denen man sich nicht versteht. Die Kommunikation mit Außenstehenden ist meist ausgeschlossen. In diesen Fällen dient UK dem Ergänzen der Lautsprache, um diese frustrierenden Situationen zu lösen und die Teilhabe in weiteren Kreisen zu ermöglichen.

Schließlich gibt es Personen, bei denen UK dem Sprachersatz dient. Hierzu zählen z.B. Menschen mit einer Körperbehinderung oder einer neurologischen Erkrankung. Oft können diese Personen zwar
Sprache „denken“, aber nicht aussprechen. Sie sind in ihrem Körper gefangen ohne sich mitzuteilen zu können. In anderen Fällen ist die Beeinträchtigung so tiefgreifend, dass auch das Verstehen von Sprache betroffen ist. Sie verstehen nicht, was wir meinen, und sind verängstigt oder aggressiv, wenn wir Dinge mit ihnen tun, die sie nicht nachvollziehen können. Hier wird UK zur Hilfe zum gegenseitigen Verstehen.

\section{Wie funktioniert UK? \\ $\nabla$}

Man nutzt körpereigene Hilfen wie Mimik, Gestik, Blicke oder Gebärden. Dies wird oft bereits instinktiv eingesetzt, hat aber seine Grenzen. Ein erster Schritt wäre, diese Formen systematisch einzusetzen z.B. ein Blinzeln für ein "Ja“ und zweimal blinzeln für ein „Nein“. Für die Nutzung von Gebärden stehen mittlerweile gute digitale Gebärdenlexika zur Verfügung, die auch außenstehenden Personen einen schnellen Zugang ermöglichen.

Des Weiteren nutzt man körperfremde Hilfen. Hier gibt es die Untergruppe der nicht elektronischen Hilfen. Hierzu gehören konkrete Gegenstände, Fotos oder Bildsymbole. Die am häufigsten in Deutschland genutzten Symbolsammlungen heißen PCS (zugehörige Software: Bordmaker) und Metacom.

Im Bereich der elektronischen Hilfen ist das Angebot sehr umfassend. Es beginnt mit einfachen Anbahnungshilfen, z.B. großen Tastern mit beweglichem Spielzeug oder Haushaltsgeräten. Hier geht es um die Erfahrung, durch Tastendruck etwas bewirken oder im Haushalt selbständig Dinge verrichten zu können.

Die nächste Stufe sind einfache Kommunikationsgeräte mit max. 32 Feldern. Die Anordnung der Felder ist festgelegt. Es werden zuvor ausgedruckte Bildkarten hinein geschoben und die Felder wie bei einem Kassettenrekorder besprochen. Drückt die beeinträchtigte Person auf ein Feld, spricht das Gerät für sie. Menschen, bei denen der Wortschatz erst langsam aufgebaut wird, finden hierin eine gute Unterstützung.

Ist das Bedürfnis zu kommunizieren weitreichender, bedarf es komplexer elektronischer Hilfen. Bei diesen Geräten kann die Oberfläche individuell angepasst werden. Sie arbeiten bild- und schriftbasiert. Einige Geräte ermöglichen zudem zu telefonieren, E-Mails zu versenden oder andere elektronische Geräte im Umfeld zu bedienen. Die Ansteuerungsmöglichkeiten reichen vom Tippen bis zur Augensteuerung. In Anlehnung an diese Hilfen gibt es mittlerweile auch Apps, die meist deutlich kostengünstiger sind. Der Nachteil liegt darin, dass sie oft kaum individuell anpassbar sind. Die Kosten für Kommunikationshilfen werden i.d.R. von der Krankenkasse auf Antrag mit ärztlichem Rezept und Gutachten übernommen.

\section{Fazit}

Unterstützte Kommunikation bietet Menschen mit komplexen Sprachbeeinträchtigungen altersunabhängig viele Hilfsmöglichkeiten. Es ist oft einfacher als man denkt. Das wichtigste aber: es ist ein Tor zu mehr Selbständigkeit, Unabhängigkeit und Selbstbestimmung.

Prof. Dr. Andrea Erdélyi, Oldenburg

überreicht durch Thieme und 